\title{
SOME NEW CONTINUITY CONCEPTS FOR METRIC PROJECTIONS
}

\author{
BY BRUNO BROSOWSKI AND FRANK DEUTSCH
}

Communicated by R. Creighton Buck, May 8, 1972

1. Introduction. There has been much recent interest in studying various continuity criteria for the set-valued metric projection onto a set $V$. Particular interest has centered around the relationship between these criteria and either the structure of the set $V$ itself or the geometry of the whole space. See, for example, [3], [4], [7], [8], [10], [16], [17], [18] and [21]. In essentially all of these papers, the concepts of lower semicontinuity (1.s.c.) and/or upper semicontinuity (u.s.c.) for set-valued mappings (as defined, for example, in Hahn [12]) played the key role.

In this note we introduce some simpler and more general "radial" continuity criteria. Roughly speaking, these criteria require that the restriction of the metric projection to certain prescribed line segments be 1.s.c. or u.s.c. It turns out that these criteria, which are formally much weaker than 1.s.c. or u.s.c., are still strong enough to generalize a number of known results, and weak enough so that many of these theorems now have valid converses (which they did not have under the stronger hypotheses of 1.s.c. or u.s.c.).

Full details of proofs along with additional results and related material will appear elsewhere.

Throughout this note $X$ will denote a (real or complex) normed linear space. For $x \in X$ and $r>0$, let

$$
S(x, r)=\{y \in X:\|x-y\|=r\} .
$$

The distance from a point $x$ to a subset $V$ of $X$ is defined by

$$
d(x, V)=\inf \{\|x-v\|: v \in V\} .
$$

The metric projection onto a subset $V$ of $X$ is the mapping $P_{V}$ which associates with each $x \in X$ its set of best approximations in $V$, i.e.

$$
P_{V}(x)=\{v \in V:\|x-v\|=d(x, V)\} .
$$

$V$ is called proximinal (resp. Chebyshev) provided $P_{V}(x)$ contains at least (resp. exactly) one point for each $x \in X . V$ is called a sun if for each $x \in X$

AMS 1970 subject classifications. Primary 41A65; Secondary 54C60.

Key words and phrases. Metric projection, best approximation, Chebyshev set, sun, set-valued mapping. 
and $v \in P_{V}(x), v \in P_{V}(v+\lambda(x-v))$ for every $\lambda \geqq 0$. The convex hull of a set $A$ is denoted $\operatorname{co}(A)$. The notation $(x, y)$ is used for the open interval $\{\lambda x+(1-\lambda) y: 0<\lambda<1\} . P_{V}$ is said to be 1.s.c. (resp. u.s.c.) at $x_{0}$ if, for open set $W$ with $W \cap P_{V}\left(x_{0}\right) \neq \varnothing$ (resp. $W \supset P_{V}\left(x_{0}\right)$ ), there exists a neighborhood $U$ of $x_{0}$ such that $W \cap P_{V}(x) \neq \varnothing$ (resp. $\left.W \supset P_{V}(x)\right)$ for every $x \in U$.

All other undefined notation or terminology can be found in [11].

2. ORL continuity. The first generalization of 1.s.c. is

Definition 2.1. Let $V \subset X$ and $x_{0} \in X . P_{V}$ is said to be outer radially lower (abbreviated ORL) continuous at $x_{0}$ if for each $v_{0} \in P_{V}\left(x_{0}\right)$ and each open set $W$ with $W \cap P_{V}\left(x_{0}\right) \neq \varnothing$, there exists a neighborhood $U$ of $x_{0}$ such that $W \cap P_{V}(x) \neq \varnothing$ for every $x$ in $U \cap\left\{v_{0}+\lambda\left(x_{0}-v_{0}\right)\right.$ : $\lambda \geqq 1\}$. $P_{V}$ is called ORL continuous if it is ORL continuous at every point.

THEOREM 2.2. Let $V \subset X$ and consider the following statements.

(1) $V$ is a sun.

(2) $P_{V}$ is $O R L$ continuous.

(3) "Local best approximations are global", i.e., for each $x \in X$, every local minimum of the function $\Phi(v)=\|x-v\|$ on $V$ is a global minimum.

(4) $V$ is a "moon" (in the sense of [1]).

Then $(1) \Rightarrow(2) \Rightarrow(3) \Rightarrow(4)$.

The implication (3) $\Rightarrow(2)$ is false in general (e.g. take $V$ to be the complement of the open unit ball in the Euclidean plane). Also, the implication $(4) \Rightarrow(3)$ is false in general. However, it is an open question whether (2) $\Rightarrow(1)$.

We call a space $X$ an MS-space if every moon in $X$ is a sun. In such a space all the conditions of Theorem 2.2 are obviously equivalent. In [1], the spaces of type $C(T), T$ compact Hausdorff (or more generally $C_{0}(T)$, $T$ locally compact Hausdorff) and $l_{1}(S)$ for any set $S$, were shown to be MS-spaces. On the negative side, no strictly convex space is a MS-space. In particular, we can state

Corollary 2.3. Let $X$ be an $M S$-space and $V \subset X$. Then $V$ is a sun if and only if $P_{V}$ is $O R L$ continuous.

It is interesting to compare this result with a particular consequence of two theorems of Vlasov ([21, Theorem 7] and [20, Theorem 13]) which establishes the hard part of the following theorem (cf. also Asplund [2] for an alternate proof):

ThEOREM. A Chebyshev set $V$ in a Hilbert space is a sun (i.e. is convex) if and only if $P_{V}$ is continuous. 
It is still unknown whether every Chebyshev set in a Hilbert space is convex. In fact, it is apparently unknown whether there exists a Chebyshev set in any space which is not a sun.

3. IRL continuity. We now give a second generalization of 1.s.c.

Definition 3.1. Let $V \subset X$ and $x_{0} \in X . P_{V}$ is said to be inner radially lower (abbreviated IRL) continuous at $x_{0}$ if, for every $v_{0} \in P_{V}\left(x_{0}\right)$ and each open set $W$ with $W \cap P_{V}\left(x_{0}\right) \neq \varnothing$, there exists a neighborhood $U$ of $x_{0}$ such that $W \cap P_{V}(x) \neq \varnothing$ for every $x$ in $U \cap\left\{v_{0}+\lambda\left(x_{0}-v_{0}\right): 0\right.$ $\leqq \lambda \leqq 1\} . P_{V}$ is called IRL continuous if it is IRL continuous at each point.

THEOREM 3.2. If $P_{V}(x)$ is convex, then $P_{V}$ is IRL continuous at $x$. In particular, if $V$ is convex or Chebyshev, $P_{V}$ is IRL continuous.

Taking $X$ to be the plane endowed with the maximum norm, $V$ to be the two point set $\left\{(1,0),\left(1, \frac{1}{2}\right)\right\}$ and $x=(0,0)$ shows that the converse of Theorem 3.2 is false.

THEOREM 3.3. Let $V \subset X$ be proximinal. Suppose that either $X$ is smooth or every convex extremal subset of $S(0,1)$ is finite-dimensional. Then, for each $x \in X \backslash V$, there exists $v \in P_{V}(x)$ such that

$$
\operatorname{co}\left(P_{V}(y)\right) \subset S(y, d(y, V))
$$

for every $y \in(x, v)$. In particular, the set $\left\{x \in X: \operatorname{co}\left(P_{V}(x)\right) \subset S(x, d(x, V))\right\}$ is dense in $X$.

Some condition like the smoothness of $X$ or the finite-dimensionality of the faces of $S(0,1)$ is essential in Theorem 3.3 as can be seen by considering $X=L_{\infty}[0,1]$ and $V=S(0,1)$.

The following three corollaries are more or less immediate consequences of Theorem 3.3.

COROLlaRY 3.4 (STEČKIN [19]). Let $V$ be a proximinal subset of a strictly convex space $X$. Then the set $\{x \in X: x$ has a unique best approximation in $V\}$ is dense in $X$.

COROllary 3.5. Let $V$ be a proximinal subset of a strictly convex space. Then $P_{V}$ is IRL continuous if and only if $V$ is Chebyshev.

In the special case when $P_{V}$ is Hausdorff continuous (resp. 1.s.c.), the "only if" part of Corollary 3.5 had been established by Blatter, Morris and Wulbert [4] (resp. Blatter [5]). It is noteworthy, however, that the converse of their results are not valid. This follows from the recent example 
of Kripke [14] of a Chebyshev subspace, having a discontinuous metric projection, in a reflexive strictly convex space.

A subset $V$ is called boundedly compact if the intersection of $V$ with every closed ball is compact.

Corollary 3.6. Let $X$ be a strictly convex and smooth Banach space and let $V \subset X$ be boundedly compact. The following are equivalent.

(1) $P_{V}$ is l.s.c.

(2) $P_{V}$ is IRL continuous.

(3) $V$ is Chebyshev.

(4) $V$ is convex.

This result follows from Corollary 3.5 and the result of Vlasov [20] that in a smooth Banach space every boundedly compact Chebyshev set is convex. The equivalence of (1), (3), and (4) had been observed earlier by Blatter, Morris and Wulbert [4].

4. ORU continuity. A generalization of u.s.c. is given by

Definition 4.1. Let $V \subset X$ and $x_{0} \in X . P_{V}$ is called outer radially upper (abbreviated ORU) continuous at $x_{0}$ if, for each $v_{0} \in P_{V}\left(x_{0}\right)$ and each open set $W \supset P_{V}\left(x_{0}\right)$, there exists a neighborhood $U$ of $x_{0}$ such that $W \supset P_{V}(x)$ for every $x$ in $U \cap\left\{v_{0}+\lambda\left(x_{0}-v_{0}\right): \lambda \geqq 1\right\}$. $P_{V}$ is called ORU continuous if it is ORU continuous at each point.

TheOREM 4.2. Let $V \subset X$ be proximinal and suppose $P_{V}(x)$ is convex for every $x \in X$. If $P_{V}$ is $O R U$ continuous, then $P_{V}(x)$ is compact for every $x \in X$.

This theorem generalizes one of Singer's [18] who had proved it in the particular case when $V$ is a subspace and $P_{V}$ is u.s.c. Theorem 4.2 is false in general if $P_{V}$ is not convex-valued. To see this, we need only take $X=l_{2}$ and $V$ to be the complement of the open unit ball. Then $P_{V}$ is ORU continuous but $P_{V}(0)=S(0,1)$ is not compact.

THEOREM 4.3. Let $V$ be a sun such that $P_{V}(x)$ is compact for every $x \in X$. Then $P_{V}$ is $O R U$ continuous.

Combining Theorems 4.2 and 4.3 we obtain

COROllary 4.4. Let $V$ be a proximinal convex set. Then $P_{V}$ is $O R U$ continuous if and only if $P_{V}(x)$ is compact for every $x$.

It is worth mentioning that Corollary 4.4 is false with u.s.c. in place of ORU continuity, even if $V$ is a subspace. This follows since there exist Chebyshev subspaces with discontinuous metric projections. (The first such example was given by Lindenstrauss $[15$, pp. 87-88].) 


\section{REFERENCES}

1. D. Amir and F. Deutsch, Suns, moons, and quasi-polyhedra, J. Approximation Theory 6 (1972), 176-201.

2. E. Asplund, Čebyšev sets in Hilbert space, Trans. Amer. Math. Soc. 144 (1969), 236-240. MR 40 \# 6238.

3. J. Blatter, Zur Stetigkeit von mengenwertigen metrischen Projektionen, Forschungsber. Landes Nordrhein-westfalen Nr. 1870, Westdeutscher Verlag, Cologne, 1967, pp. 17-38. MR 36 \# 3033.

4. J. Blatter, P. D. Morris and D. Wulbert, Continuity of the set-valued metric projection, Math. Ann. 178 (1968), 12-24. MR 37 \#4563.

5. J. Blatter, Weiteste Punkte und nächste Punkte, Rev. Roumaine Math. Pures Appl. 14 (1969), 615-621. MR 40 \# 4737.

6. B. Brosowski and R. Wegmann, Charakterisierung bester Approximationen in normierten Vektorräumen, J. Approximation Theory 3 (1970), 369-397. MR 43 \# 3713.

7. - On the lower semi-continuity of the set-valued metric projection, J. Approximation Theory (to appear).

8. B. Brosowski, Über eine Fixpunkteigenschaft der metrischen Projektion, Computing 5 (1970), 295-302.

9. B. Brosowski and F. Deutsch, On some geometrical properties of suns, J. Approximation Theory (to appear).

10. A. L. Brown, Best n-dimensional approximation to sets of functions, Proc. London Math. Soc. (3) 14 (1964), 577-594. MR 29 \#5033.

11. N. Dunford and J. T. Schwartz, Linear operators. I: General theory, Pure and Appl. Math., vol. 7, Interscience, New York, 1958. MR 22 \# 8302.

12. H. Hahn, Reelle Funktionen, Akademie Verlagsgesellschaft, Leipzig, 1932; reprint, Chelsea, New York, 1948.

13. V. Klee, Remarks on nearest points in normed linear spaces, Proc. Colloq. on Convexity (Copenhagen, 1965), Københavns Univ. Mat. Inst., Copenhagen, 1967, pp. 168-176. MR 36 \# 6906.

14. B. Kripke, Unpublished.

15. J. Lindenstrauss, Extension of compact operators, Mem. Amer. Math. Soc. No. 48 (1964), 112 pp. MR 31 \# 3828.

16. P. D. Morris, Metric projections onto subspaces of finite codimension, Duke Math. J. 35 (1968), 799-808. MR 38 \# 1503.

17. E. V. Osman, Continuity of metric projection and some geometric properties of the unit sphere in a Banach space, Dokl. Akad. Nauk SSSR 185 (1969), 34-36= Soviet Math. Dokl. 10 (1969), 291-293. MR 39 \# 7396.

18. I. Singer, On set-valued metric projections, Proc. Conf. on Linear Operators and Approximation Theory (Oberwolfach, 1971) (to appear).

19. S. B. Stečkin, Approximation properties of sets in normed linear spaces, Rev. Math. Pures Appl. 8 (1963), 5-18. (Russian) MR 27 \# 5108.

20. L. P. Vlasov, Approximatively convex sets in Banach spaces, Dokl. Akad. Nauk SSSR 163 (1965), 18-21 = Soviet Math. Dokl. 6 (1965), 876-879. MR 34 \#6497.

21. - C Cebyšev sets and approximatively convex sets, Mat. Zametki 2 (1967), 191200. (Russian) MR 35 \# 5904.

Department of Mathematics, Pennsylvania State University, University Park, PENNSYlVANIA 16802

Current address (Bruno Brosowski): Gesellschaft für wissenschaftliche Datenverarbeitung mbH, 34 Göttingen, Am Fassberg, Federal Republic of Germany. 